\title{
Inversión ecoeficiente: efectos sobre el desempeño económico y financiero de los grupos empresariales del Dow Jones Sustainability World Index
}

\author{
Esteban Pérez-Calderón ${ }^{a}$, María Pache-Durán ${ }^{\mathrm{b}}$, Patricia Milanés-Montero ${ }^{\mathrm{c}}$ \\ $a, b, c)$ Department of Finance and Accounting, University of Extremadura, Av. de Elvas, s/n, 06006, Badajoz-Spain.
}

${ }^{a}$ Corresponding author.

E-mail address: estperez@unex.es

\section{A R T I C LE IN F O}

\section{Article history:}

Received 15 November 2019

Accepted 25 February 2020

Available online 1 July 2021

\section{Códigos JEL:}

M14

M21

Q56

Palabras clave

Ecoeficiencia

Inversión ecoeficiente

Dow Jones Sustainability Index

Carbon Disclosure Project

\section{R E S U M E N}

El impacto de la actividad económica sobre el medio ambiente es innegable, causando daños irreparables. Por lo anterior, la inversión en eficiencia en emisiones es una de las soluciones al problema. En la revisión de la literatura se observan efectos dispares entre la inversión asociada a comportamientos ecoeficientes y la rentabilidad económica-financiera de las empresas. El objetivo del estudio fue aportar mayor evidencia empírica sobre estos efectos. Se utilizó un panel de datos balanceado para una muestra de 57 grupos empresariales, líderes europeos cotizados, para los años 2012-2015. Los resultados desvelan una relación positiva entre los niveles de ecoeficiencia y el desempeño económico-financiero. Las recompensas alcanzadas por los grupos empresariales, por la mejora de sus resultados y a través de los mercados financieros, deberían facilitar la aplicación de normativas con el objetivo de reducir el impacto de la actividad empresarial sobre el medioambiente.

(C)2021 ASEPUC. Published by EDITUM - Universidad de Murcia. This is an open access article under the CC BY-NC-ND license (http://creativecommons.org/licenses/by-nc-nd/4.0/).

Eco-efficient investment: effects on the economic and financial performance of Dow Jones Sustainability World Index companies

A B S T R A C T

The impact of economic activity on the environment is undeniable, causing irreparable damage. Thus, investment in emission efficiency is one of the solutions to the problem. A review of the literature shows disparate effects between investment associated with eco-efficient behaviour and the economic-financial profitability of companies. The aim of the study was to provide more empirical evidence on these effects. A balanced data panel was used for a sample of 57 large groups of listed companies for the years 2012-2015. The results reveal a positive relationship between eco-efficiency levels and economic-financial performance. The rewards achieved by companies for improving their results and through financial markets should facilitate the application of regulations with the aim of reducing the impact of business activity on the environment.

(C2021 ASEPUC. Publicado por EDITUM - Universidad de Murcia. Este es un artículo Open Access bajo la licencia CC BY-NC-ND (http://creativecommons.org/licenses/by-nc-nd/4.0/). 


\section{Introducción}

Desde la firma del protocolo de Kyoto en 1997, se ha tomado especial conciencia de los efectos de la actividad empresarial sobre el cambio climático. Los desastres naturales como tormentas devastadoras, el deshielo de los polos, inundaciones o las sequías extremas, se han asociado a los efectos de las emisiones de gases efecto invernadero (GEI) sobre las capas de nuestra atmósfera y el incremento medio de las temperaturas en todo el planeta (Hallegatte et al., 2016; IPCC, 2018). $\mathrm{El}$ control de las emisiones de GEI, en particular de $\mathrm{CO}_{2}$, es uno de los frentes clave para combatir el cambio climático. Los principales países y empresas de todo el mundo han ido adquiriendo compromisos de reducción de sus emisiones de GEI (UNFCCC, 2016). Así, las empresas están desarrollando estrategias de actuaciones ecoeficientes con el objetivo de gestionar sus emisiones pero sin descuidar la repercusión que puedan tener estos comportamientos en su rentabilidad o valoración financiera.

El término de ecoeficiencia comienza a utilizarse en la década de los setenta como "eficiencia medioambiental" (Freeman, Myrick \& Haveman, 1973). La consolidación y el desarrollo del concepto de ecoeficiencia la lleva a cabo el Consejo Empresarial para el Desarrollo Sostenible en la Cumbre Mundial de Desarrollo Sostenible celebrada en Río de Janeiro en 1992. Schmidheiny (1992) fue el primero en proponer un cambio de percepción de los sectores industriales ante el problema del cambio climático. Este autor reconoce la responsabilidad de las empresas en la solución que pueden dar al problema en lugar de responsabilizarles del mismo.

La satisfacción de las expectativas de la sociedad y del resto de stakeholders de las organizaciones por los efectos de la actividad empresarial sobre el cambio climático debe conjugarse con la necesaria viabilidad económica y financiera, surgiendo el concepto de ecoeficiencia empresarial. En la revisión de la literatura previa nos encontramos con estudios que obtienen resultados divergentes en la relación que se observa entre el desempeño económico (DEc), el desempeño financiero (DFn) y la eficiencia de las empresas en emisiones (Gallego-Álvarez, Rodríguez-Domínguez \& García-Sánchez, 2011; Yang \& Zhang, 2018). También, se encuentran trabajos que demuestran una relación positiva (King \& Lenox, 2001; Ittner, Larcker \& Randall, 2003; Al-Tuwaijri, Christensen \& Hughes, 2004; Nakao et al., 2007; Rokhmawati, Sathye \& Sathye, 2015). Sin embargo, en otros estudios, se obtienen resultados contrarios o inconcluyentes (Lothe, Myrtveit \& Trapani, 1999; Wagner, 2005; Sueyoshi \& Goto, 2009; Rassier \& Earnhart, 2010; Arbelo, Pérez-Gómez, Rosa-González \& Ramos, 2014).

En línea con lo anterior, la teoría de la ecoeficiencia está muy relacionada con el concepto de innovación sostenible. Las inversiones que lleve a cabo la empresa deben atender a la viabilidad financiera de la misma, al mismo tiempo que deberá reducir su impacto sobre el medioambiente (Kemp, 1997; Adams et al., 2016). El impacto de la actividad empresarial en el medioambiente está muy condicionado por la tecnología que aplican las organizaciones en sus procesos de producción (Teece, 2010; Kneller \& Manderson, 2012; Przychodzen \& Przychodzen, 2015). Así pues, sería interesante conocer hasta qué punto les resulta beneficioso a las empresas invertir en nuevas tecnologías o estructuras de producción que supongan una menor emisión de GEI.

En este contexto, la preocupación de las empresas por la ecoeficiencia en la última década ha ido incrementándose pero resulta insuficiente. Tanto el nivel de inversión como la divulgación de información sobre sus actuacio- nes ecoeficientes han estado en función del tamaño de las empresas o del sector de actividad (Camisón-Zornoza, Lapiedra-Alcamí, Segarra-Ciprés \& Boronat-Navarro, 2004; Barbu, Dumontier, Feleag, \& Feleag, 2014; Prado-Lorenzo, Rodriguez-Dominguez, Gallego-Álvarez \& García-Sánchez, 2009; González-Benito \& González-Benito, 2006).

La finalidad del presente trabajo consiste en el análisis de los comportamientos ecoeficientes y sus efectos sobre el DEc y DFn de las empresas. Más concretamente, los autores estudian los efectos de las inversiones en ecoeficiencia sobre la rentabilidad económica-financiera y el valor de la empresa. La muestra está compuesta por 57 grupos empresariales, líderes europeos en sus sectores, en cuanto a divulgación de información sobre responsabilidad medioambiental. La metodología de análisis econométrico ha sido la técnica de panel de datos.

El objetivo del trabajo es, por tanto, analizar la relación entre las inversiones de los grupos empresariales analizados en actuaciones ecoeficientes y:

- las ratios de eficiencia en emisiones de gases. Se espera una relación positiva.

- el resultado económico-financiero. La relación se espera positiva, a mayor beneficio en el ejercicio mayor disposición a invertir en medidas de protección medioambiental.

- el valor alcanzado en los mercados de capitales. La relación se espera positiva, por la mejora esperada de beneficio extra obtenido. Además de la mejora de la imagen del grupo ante sus stakeholders gracias a su comportamiento responsable ante el cambio climático.

Los resultados muestran que los grupos empresariales más proactivos, con actuaciones medioambientales que conllevan mejores índices de ecoeficiencia, en términos de emisiones directas, son los que mejor DEc y DFn consiguen. Aunque, para el periodo y la muestra analizados, no resulte ser premiada por los mercados de valores. Se puede afirmar, por tanto, una relación positiva entre los niveles de ecoeficiencia y el rendimiento económico-financiero, siendo negativa con la valoración hecha por el mercado.

Así, el estudio contribuye aumentando la evidencia empírica de la relación que se establece entre los comportamientos ecoeficientes (ahorros e inversiones ecoeficientes), y el DEc y DFn de los grupos de empresa similares a los analizados. Estos resultados sirven de referencia para el resto de grupos empresariales y justificarán futuras inversiones en actuaciones ecoeficientes que repercutirán en un menor impacto de la actividad empresarial sobre el medioambiente. Además, las instituciones responsables de la regulación medioambiental contarán con una mayor evidencia empírica en cuanto a los efectos de las inversiones en comportamientos ecoeficientes y el desempeño empresarial.

Adicionalmente, la contribución del estudio se encuentra en el empleo de una variable representativa de la inversión en ecoeficiencia. En gran parte de las investigaciones previas se han empleado los gastos de I+D como proxy a las inversiones llevadas a cabo en medidas de protección medioambiental. La mayor limitación de esta variable sería que, además de innovaciones cuyo objetivo es minorar el impacto en el medioambiente, esa inversión puede estar atendiendo a otros objetivos muy distintos. Así pues, en este trabajo se ha utilizado una variable que representa directamente a las inversiones destinadas a actuaciones medioambientales. Esta variable ha sido recopilada de los cuestionarios consultados en la plataforma del Carbon Disclosure Project (CDP). 
El trabajo se estructura como sigue. Tras la presente introducción, en la segunda sección, se lleva a cabo una revisión de la literatura de los conceptos y las relaciones referidos al desempeño medioambiental, en términos de ecoeficiencia, y el desempeño económico-financiero. Esta segunda sección finaliza con el planteamiento de las hipótesis de trabajo. A continuación, en la sección tercera, se expone la muestra y metodología de investigación empleada y, finalmente, se comentan los resultados y las conclusiones del estudio, en las secciones cuarta y quinta, respectivamente.

\section{Revisión de la literatura previa. Diseño de las hipó- tesis de trabajo}

En las últimas décadas, el mundo empresarial ha asumido su responsabilidad social y medioambiental respondiendo a las demandas de comportamientos esperados por parte de sus stakeholders (Miroshnychenko, Barontini \& Testa, 2017; Stubbs \& Cocklin, 2008; Pomering \& Johnson, 2009; Sprinkle \& Maines, 2010; Weinhofer \& Hoffmann, 2010). En la literatura previa ha quedado demostrado el efecto positivo de un comportamiento socialmente responsable sobre la rentabilidad de la empresa, satisfacción de sus clientes, coste de endeudarse o la mejora de su marca y reputación (Lankoski, 2006; Wagner, 2015; Knox \& Maklan, 2004; Bendixen \& Abratt, 2007; La Rosa, Liberatore, Mazzi \& Terzani, 2018).

Respecto a la responsabilidad social corporativa siempre se ha discutido hasta qué punto sería conveniente su regulación. Para el caso particular de la responsabilidad medioambiental, se han llevado a cabo algunas iniciativas que, aunque en sus fases iniciales las empresas se podían ajustar voluntariamente a lo exigido, finalizan con la implantación de un sistema cuota-mercado que consiste en la asignación de cuotas de emisiones que las empresas no pueden superar o en otro caso serían penalizadas, permitiéndose el mercadeo (EU, 2009; ICCT, 2017). Este tipo de iniciativas están siendo tomadas como referencia por otras zonas y gobiernos para el control de las emisiones a la atmósfera por parte de las grandes empresas y, en particular, en los países desarrollados y para determinados sectores de actividad. Por todo ello, en los últimos años está aumentando el número de empresas que han ido actuando de manera voluntaria para conseguir reducir sus emisiones. De este modo, esas empresas se anticipan a una posible regulación más estricta y extensiva que pueda afectarlas en un futuro más o menos inmediato (Blanco, Caro \& Corbett, 2016).

Así pues, ante esta presión legislativa latente y la generada por sus stakeholders, las grandes empresas se están adaptando a las expectativas sobre su comportamiento ante el cambio climático realizando inversiones en tecnologías y bienes de producción que provoquen reducciones en sus emisiones. Dependiendo de los sectores y su impacto sobre el medioambiente, el nivel de ajuste ha sido mayor o menor pero las empresas enfocan sus actuaciones, sobre todo, en la reducción de emisiones de $\mathrm{CO}_{2}$. Entre las principales medidas para conseguir reducir las emisiones de GHG estaría la sustitución de fuentes de energía, la inversión en nuevas tecnologías de producción que mejoran la combustión de las fuentes de energía y otras de bajo consumo energético (Majumdar \& Marcus, 2001; Rubashkina, Galeotti \& Verdolini, 2015).

Según lo anterior, las empresas deben generar capacidades y habilidades para reducir sus emisiones de gases y, al mismo tiempo, mantener sus beneficios económicos y financieros. A esa habilidad se refiere la Hipótesis de Porter que define una estrategia doblemente ganadora para la empresa y la socie- dad (Porter, 1991). El planteamiento y los trabajos de Porter manifiestan la oportunidad que supone para algunas empresas una actuación responsable con el medioambiente, ya que si se adaptan a las exigencias de reducir su impacto medioambiental conseguirán ser más competitivas y mejorar su DEc y su DFn (Porter y Van der Linde, 1995a, 1995b). En este sentido, es interesante estudiar si las empresas que invierten en un comportamiento ecoeficiente consiguen mantener sus rentabilidades y al mismo tiempo reducen sus emisiones.

Por otro lado, las empresas deben atender a las demandas de sus stakeholders quienes se muestran mucho más sensibles con aquellas actuaciones que estén asociadas al cambio climático. Así, las empresas visualizan su comportamiento respecto a la protección medioambiental participando en proyectos de alcance internacional como el CDP, formando parte de exigentes índices de valores como el Dow Jones Sustainability World Index (DJSWI), además de publicar un gran volumen de información sobre su compromiso y actuación medioambiental en sus portales web e informes medioambientales (Blanco et al., 2016; Pérez-Calderón, Milanés-Montero \& Ortega-Rossell, 2012; Ziegler \& Schröder, 2010).

Por cuanto a la medición de la ecoeficiencia se refiere, en los estudios previos, se ha asociado a la relación entre el impacto medioambiental generado y la producción obtenida. Esto es, el consumo energético o la emisión generada por unidad de producción, inversión o beneficio obtenidos. Esta ratio de ecoeficiencia ha sido analizada por muchos estudios respecto a niveles de innovación, rentabilidades económicas o incremento del valor de la empresa en los mercados de capitales (WBCSD, 2000; Verfaillie \& Bidwell, 2000; Müller \& Sturm, 2001; Huppes \& Ishikawa, 2005; Molina-Azorín, Claver-Cortés, López-Gamero \& Tarí, 2009).

En los estudios analizados en la literatura previa, se ha comprobado que no se distingue entre DEc y DFn. Generalmente, se ha hecho referencia al segundo término para analizar los efectos del desempeño medioambiental sobre variables de rentabilidades económico-financieras como la rentabilidad sobre activos, la rentabilidad sobre recursos propios o el margen sobre el beneficio. En otros estudios se han empleado variables representativas de la valoración que realiza el mercado de valores de estos comportamientos medioambientalmente responsables, estas son, $\mathrm{Q}$ de Tobin, precio de las acciones o valoración en el mercado de capitales (ver Tabla 1).

En este trabajo se distingue entre DEc y DFn, cuando analizamos los efectos del desempeño medioambiental.

En el primer caso, con DEc, nos referimos a la mejora que puede provocar la ecoeficiencia en la rentabilidad de la empresa dependiente de los resultados de su actividad. Ya que el resultado final del ejercicio o beneficio neto engloba otra serie de conceptos que no están relacionados directamente con el corazón de la actividad de la empresa. También hay que tener en cuenta que el resultado económico está siendo condicionado por otras variables a las que afecta un buen comportamiento medioambiental como pueden ser: el incremento de clientes por mejora de imagen del producto o empresa; disminución de costes por conseguir innovaciones en procesos, rutinas u optimización en materias primas empleadas; reducción de costes de financiación; mejora de productividad, entre otras. Según lo anterior, podemos establecer como primera hipótesis de trabajo que:

H1: los grupos empresariales que invierten en mayor medida en actuaciones ecoeficientes obtienen una mayor rentabilidad económica (ROA). 
Tabla 1

Estudios de la relación entre la inversión ecoeficiente y el desempeño económico-financiero

\begin{tabular}{|c|c|c|}
\hline Autor/Año & Muestra & Resultados \\
\hline $\begin{array}{l}\text { García-Sánchez et al. } \\
\text { (2019) }\end{array}$ & $\begin{array}{l}6454 \text { empresas internacio- } \\
\text { nales. 2002-2017. }\end{array}$ & $\begin{array}{l}\text { Ecoinnovación y ecodiseño tienen efecto negativo sobre ROA } \\
\text { y ROE en el corto plazo, por los altos costes que suponen. El } \\
\text { mercado de valores premia esta proactividad de protección } \\
\text { medioambiental (Q de Tobin). }\end{array}$ \\
\hline $\begin{array}{l}\text { Przychodzen \& Przychod- } \\
\text { zen (2015) }\end{array}$ & $\begin{array}{l}\text { Compañías del IBEX35. Es- } \\
\text { paña. 2010-2015. }\end{array}$ & $\begin{array}{l}\text { Relación positiva entre las prácticas en innovación sostenible y } \\
\text { la rentabilidad económica, medida por el margen de beneficio } \\
\text { neto. }\end{array}$ \\
\hline $\begin{array}{l}\text { Przychodzen \& Przychod- } \\
\text { zen (2015) }\end{array}$ & $\begin{array}{l}\text { Compañías del IBEX35. Es- } \\
\text { paña. 2010-2015. }\end{array}$ & $\begin{array}{l}\text { Relación positiva entre las prácticas en innovación sostenible y } \\
\text { la rentabilidad económica, medida por el margen de beneficio } \\
\text { neto. }\end{array}$ \\
\hline $\begin{array}{l}\text { Nishitani, Kaneko, Fujii \& } \\
\text { Komatsu (2011) }\end{array}$ & $\begin{array}{l}426 \text { empresas japonesas. } \\
2002-2008 .\end{array}$ & $\begin{array}{l}\text { Un enfoque de prevención conlleva ventajas competitivas que } \\
\text { incrementan la demanda y mejoras en la productividad. }\end{array}$ \\
\hline $\begin{array}{l}\text { Miroshnychenko et al. } \\
\text { (2017) }\end{array}$ & $\begin{array}{l}3490 \text { empresas. } 58 \text { países } \\
\text { distintos. 2002-2014. }\end{array}$ & $\begin{array}{l}\text { Aquellas empresas que invierten en mecanismos de preven- } \\
\text { ción de polución y gestionan su cadena de suministro consi- } \\
\text { guen una mejor desempeño económico-financiero en términos } \\
\text { de ROE, ROA y Return on Sales (ROS). }\end{array}$ \\
\hline Hart \& Ahuja (1996) & $\begin{array}{l}127 \text { empresas de EE.UU. } \\
\text { S\&P 500. 1989-92. }\end{array}$ & $\begin{array}{l}\text { Efecto positivo a corto plazo. Las variables ROA y ROS se ven } \\
\text { afectadas positivamente en los dos primeros años. La variable } \\
\text { ROE tarda más tiempo. }\end{array}$ \\
\hline Al-Tuwaijri et al. (2004) & $\begin{array}{l}198 \text { empresas de EE.UU. } \\
\text { S\&P 500. } 1994\end{array}$ & $\begin{array}{l}\text { Una relación positiva entre el desempeño financiero y el } \\
\text { desempeño ambiental, en línea con el trabajo de Porter y Van } \\
\text { der Linde (1995a, 1995b). }\end{array}$ \\
\hline Iwata \& Okada (2011) & $\begin{array}{l}268 \text { empresas japonesas } \\
\text { manufactureras. } \\
2008 .\end{array}$ & $\begin{array}{l}\text { Relación positiva entre la reducción de emisiones de gases y el } \\
\text { desempeño financiero medido por ROE, ROA, ROS, ROI (Re- } \\
\text { turn on Investment), ROIC (Return on Invested Capital) y To- } \\
\text { bin's Q. }\end{array}$ \\
\hline King \& Lenox (2002) & $\begin{array}{l}614 \text { Empresas manu- } \\
\text { factureras de EE.UU. } \\
\text { 1991-1996. }\end{array}$ & $\begin{array}{l}\text { Efecto positivo del desempeño medioambiental sobre el } \\
\text { desempeño financiero, pero no para todas las especificaciones }\end{array}$ \\
\hline Misani \& Pogutz (2015) & $\begin{array}{l}127 \text { compañías CDP. 2007- } \\
2013 .\end{array}$ & $\begin{array}{l}\text { Relación positiva entre gestión de emisiones y la rentabilidad } \\
\text { financiera medida por la Tobin's } Q\end{array}$ \\
\hline $\begin{array}{l}\text { Feldman, Soyka \& Ameer } \\
\text { (1997) }\end{array}$ & $\begin{array}{l}300 \text { empresas públicas de } \\
\text { EE.UU. 1980-87 y 1988-94, } \\
\text { indexadas en Standard \& } \\
\text { Poor's }\end{array}$ & $\begin{array}{l}\text { La implementación de un Sistema de Gestión Ambiental } \\
\text { (SGA), evidencias de progreso hacia la reducción de la ge- } \\
\text { neración de contaminantes y la minimización de riesgos. Las } \\
\text { compañías que mejoran en sus sistemas de gestión medioam- } \\
\text { biental incrementan la riqueza de sus inversores hasta en un } \\
5 \% \text {. }\end{array}$ \\
\hline Butz \& Plattner (1999) & $\begin{array}{l}65 \text { empresas europeas. } \\
1996-1997\end{array}$ & $\begin{array}{l}\text { Relación positiva entre las empresas con alta calificación en } \\
\text { comportamiento de responsabilidad medioambiental y la ren- } \\
\text { tabilidad obtenida por sus accionistas. }\end{array}$ \\
\hline Wagner (2005) & $\begin{array}{l}\text { Empresas europeas de la } \\
\text { Industria del papel. 1995- } \\
1997\end{array}$ & $\begin{array}{l}\text { Relación negativa entre un índice de emisiones de gases efecto } \\
\text { invernadero y el desempeño financiero. Estos resultados son } \\
\text { puntualizados para las empresas con una mayor orientación } \\
\text { de protección medioambiental. Los desempeños financieros y } \\
\text { medioambiental están relacionadas con la calidad de la ges- } \\
\text { tión. }\end{array}$ \\
\hline Thomas (2001) & $\begin{array}{l}422 \text { empresas. Gran Breta- } \\
\text { ña, 1985-1997 }\end{array}$ & $\begin{array}{l}\text { Relación significativa y negativa entre el índice medioambien- } \\
\text { tal y el rendimiento económico. }\end{array}$ \\
\hline
\end{tabular}
tal y el rendimiento económico.

Por otro lado, el DFn sería la retribución que recibe el accionista por la obtención de beneficio neto al final del ejercicio y también la revalorización del valor de la empresa en los mercados de capitales (Valor bursátil). Comenzando con la variable de la rentabilidad financiera, como se ha visto, en la revisión de la literatura ha sido comúnmente utilizada. A diferencia de la rentabilidad económica, el resultado que se utiliza para su cálculo incluye, sobre todo, partidas referidas a la financiación de la empresa. Así, se pasa de una visión sobre la eficiencia operacional, como la que nos da el ROE, a otra más global en la que se han incluido todos los resultados el ejercicio, en particular los financieros. Por lo anterior, nos interesa comprobar la relación de la ecoeficiencia con la rentabilidad financiera, postulando para ello la segunda hipótesis:

H2: los grupos empresariales que invierten en mayor medida en actuaciones ecoeficientes obtienen una mayor rentabilidad financiera (ROE) 
Por último, como igualmente se ha justificado en la revisión de la literatura, los mercados de capitales, además de invertir en la empresa por su rentabilidad económicafinanciera, pueden reconocer oportunidades de futuro y también premiar el compromiso o proactividad respecto al impacto de la actividad empresarial sobre el medioambiente. Es más, si un grupo empresarial quiere optar a ser incluido en un índice tan selectivo como el DJSWI deberá cumplir con unas exigencias en cuanto a inversiones, comportamientos, transparencia y divulgación de información. Resulta interesante comprobar si la ecoeficiencia está siendo recompensada por los accionistas. Para ello planteamos la siguiente hipótesis:

H3: los grupos empresariales que invierten en mayor medida en actuaciones ecoeficientes se ven recompensados por el mercado de valores (QT)

\section{Metodología de investigación}

\subsection{Muestra y variables}

El panel consta de 228 observaciones, 57 grupos empresariales líderes por un período de 4 años (2012-2015). Los datos financieros se obtuvieron de la base de datos ORBIS y los indicadores medioambientales de los informes publicados por el CDP (véase el Apéndice A). Estas empresas son los referentes en el mundo por sus actividades de responsabilidad social corporativa, especialmente en lo que se refiere al medio ambiente.

La literatura previa analiza la relación entre distintas variables para el análisis del desempeño ambiental y el desempeño económico y financiero. Un gran número de artículos toman el retorno sobre el activo (ROA) como variable dependiente para representar la rentabilidad económica de la empresa (Hart \& Ahuja, 1996; Russo \& Fouts, 1997; King \& Lenox, 2002; Aragón-Correa, Hurtado-Torres, Sharma y García-Morales, 2008; Iwata \& Okada, 2011; Rokhmawati et al., 2015; Miroshnychenko et al., 2017). Para medir la rentabilidad financiera se repiten los estudios que utilizan la variable retorno sobre el patrimonio (ROE); así lo hicieron autores como Fombrun \& Shanley (1990), Wagner, Van Phu, Azomahou \& Wehrmeyer (2002), Iwata \& Okada (2011) y Miroshnychenko et al. (2017). Como medida de rentabilidad financiera en términos de revalorización de las acciones, generalmente, se ha empleado la Tobin's Q (King \& Lenox, 2001 y 2002; Horváthová, 2010; Iwata \& Okada, 2011; Misani \& Pogutz, 2015).

En este estudio, como variables de referencia para la medida del desempeño financiero se utilizan el Retorno sobre el Patrimonio (ROE) y el valor de mercado de la empresa relativizado con los Fondos Propios (QT). La medida de rentabilidad económica es la ROA. Las variables asociadas a las inversiones en ecoeficiencia son las inversiones declaradas por los grupos empresariales en los informes publicados en el CDP. Estas inversiones se encuentran clasificadas según su tiempo de aplicación, a saber: corto, medio y largo plazo (EIST, EIMT, EILT). En el caso de esta variable no se han encontrado estudios que la hayan empleado.

Los datos relativos a las emisiones a la atmósfera (emisiones netas en toneladas métricas de $\mathrm{CO}_{2}$ ) están relativizados respectos a las cifras de ventas de ese año y se utilizan como variables asociadas a la ecoeficiencia (ES). Esta variable ya había sido utilizada en los trabajos de Hatakeda, Kokubu, Kajiwara \& Nishitani (2012) y Wagner (2005), así como en otros trabajos más recientes de Delmas \& Nairn-Birch
(2010), Busch \& Hoffmann (2011), Rahman, Rasid \& Basiruddin (2014), Gallardo-Álvarez \& Castilla-Polo (2015).

En el análisis de la valoración de los grupos empresariales por el mercado de valores se incluye una variable referida a la divulgación de información respecto al impacto medioambiental (DisCDP). Esta transparencia y comunicación de información referida a la responsabilidad medioambiental de la empresa condiciona la revalorización de la compañía como se ha demostrado en estudios como los de Al-Tuwaijri et al. (2004).

Las variables de control se seleccionaron a partir de la revisión de la literatura siendo las que se utilizan generalmente en los estudios de ecoeficiencia. Las variables de control son el Activo Total (TA) y el Gasto en Investigación y Desarrollo (RD), que son indicativas de la medida en que la compañía está optando por el desarrollo de nuevos productos, rutinas de trabajo o cualquier innovación como aproximación a su compromiso de adaptación continua a nuevos retos que pueden estar asociadas a comportamientos ecoeficientes (Iwata \& Okada, 2011; Miroshnychenko et al., 2017; García-Sánchez et al., 2019).

\subsection{Estadísticos y modelos de regresión}

Como se ha visto, la literatura previa sobre la relación entre el DEc y el DFn ha dado lugar no sólo a resultados favorables y desfavorables, sino también a resultados no concluyentes. Con el fin de aportar una mayor evidencia empírica en lo que se refiere a esta relación, vamos a centrarnos en variables que muestren de forma más directa los efectos en el ambiente, y hacerlo desde la perspectiva de los inversores, ya que ellos son el referente financiero por excelencia, vamos a comparar nuestra hipótesis de trabajo con los siguientes modelos:

$$
\begin{aligned}
& R O A_{i t}=\beta_{0}+\beta_{1} E S_{i t}+\beta_{2} I_{i t}+\varnothing X_{i t}+\varepsilon_{i j} \quad \text { (Modelo1) } \\
& R O E_{i t}=\beta_{0}+\beta_{1} E S_{i t}+\beta_{2} I_{i t}+\varnothing X_{i t}+\varepsilon_{i j} \quad \text { (Modelo2) } \\
& Q T_{i t}=\beta_{0}+\beta_{1} E S_{i t}+\beta_{2} I_{i t}+\varnothing X_{i t}+\varepsilon_{i j} \quad \text { (Modelo3) }
\end{aligned}
$$

Donde, $\mathrm{X}_{\mathrm{it}}=\mathrm{TA} A_{\mathrm{it}}$

Para el Modelo (3), las variables $R O E_{\text {it }}, D i s C D P_{\text {it }}$ y $R D_{\text {it }}$ fueron añadidas.

La descripción de las variables sería la siguiente:

$i$ se refiere a cada empresa, $t$ al año.

$R O A_{i t}$, Rentabilidad Económica; relación de rentabilidad respecto a los activos totales.

$R O E_{i t}$, Rentabilidad financiera; relación entre el beneficio antes de impuestos y el patrimonio neto.

$Q T_{i t}$, valor de mercado de la empresa en relación con el volumen de EBITDA (Resultado Bruto de Explotación).

$E S_{i t}$, emisiones directas de GEI medidas en toneladas métricas equivalentes de $\mathrm{CO}_{2}$. Esta variable se relativiza con las ventas del periodo ( $\mathrm{Tn} \mathrm{CO}_{2}$ e por Euro de facturación).

$E I_{i t}$, Inversión en medidas para reducir el impacto en el medioambiente. Esta variable se relativiza con las ventas del periodo (Euros de inversión por Euro de Ingresos). Se han utilizado tres períodos de tiempo: corto, medio y largo plazo. 
Tabla 2

Estadísticos Descriptivos del desempeño económico-financiero

\begin{tabular}{lrrrr}
\hline \multicolumn{1}{c}{ Variable } & \multicolumn{1}{c}{ Media } & Desv. Típica & \multicolumn{1}{c}{ Mínimo } & \multicolumn{1}{c}{ Máximo } \\
\hline$R O A$ & 5.8899 & 4.2526 & -5.772 & 19.355 \\
$R O E$ & 16.2997 & 13.3233 & -22.827 & 85.087 \\
$T A(000 €)$ & $43,044,852.29$ & $46,318,255.09$ & $1,731,771.86$ & $215,908,100.00$ \\
$Q T$ & 9.7779 & 4.3843 & 3.02 & 24.24 \\
$R D$ & 2.3427 & 3.9931 & 0 & 19.89 \\
$E S(T n)$ & $6,133,439.63$ & 295,00 & $18.562,53$ & $127,801,261.00$ \\
$E I S T(000 €)$ & $4.488,69$ & $18.688,53$ & 902,00 & $159.784,49$ \\
$E I M T(000 €)$ & $39.558,05$ & $234.621,30$ & $14.400,00$ & $2.216 .000,00$ \\
$E I L T(000 €)$ & 283,94 & $920.406,24$ & 0,49 & $6.828 .100,00$ \\
\hline
\end{tabular}

Fuente: Elaboración Propia

Tabla 3

Análisis de correlaciones. Coeficiente de Pearson

\begin{tabular}{|c|c|c|c|c|c|c|c|c|c|c|}
\hline & $T A$ & $R D$ & $R O E$ & $R O A$ & $Q T$ & $E S$ & DisCDP & IEST & IRMT & IRLT \\
\hline$T A$ & 1,0000 & & & & & & & & & \\
\hline$R D$ & 0,0531 & 1,0000 & & & & & & & & \\
\hline$R O E$ & 0,0380 & 0,0799 & 1,0000 & & & & & & & \\
\hline$R O A$ & $-0,1363 *$ & 0,0894 & $0,7876^{*}$ & 1,0000 & & & & & & \\
\hline$Q T$ & $-0,4152 *$ & $0,2226^{*}$ & $-0,0281$ & $0,1955^{*}$ & 1,0000 & & & & & \\
\hline$E S$ & $0,2799 *$ & $-0,3359 *$ & $-0,1115$ & $-0,1784 *$ & $-0,2706 *$ & 1,0000 & & & & \\
\hline DisCDP & $0,3310 *$ & 0,1304 & 0,1381 & 0,1302 & $-0,0458$ & 0,0566 & 1,0000 & & & \\
\hline$I R S T$ & 0,1927 & $-0,1599$ & $-0,2202 *$ & $-0,1746$ & $-0,1960$ & $0,3613^{*}$ & $-0,0040$ & 1,0000 & & \\
\hline$I R M T$ & $0,2311 *$ & 0,0946 & $-0,1603$ & $-0,2408 *$ & $-0,0860$ & $0,2482^{*}$ & 0,1031 & $0,2795 *$ & 1,0000 & \\
\hline$I R L T$ & $0,4543^{*}$ & $-0,0521$ & $-0,1068$ & $-0,2408^{*}$ & $-0,1421$ & $0,4466 *$ & 0,0158 & $0,3789 *$ & $0,4363 *$ & 1,0000 \\
\hline
\end{tabular}

Fuente: Elaboración Propia

$T A_{i t}$, activos totales (logaritmo de los activos totales).

$R D_{i t}$, investigación y desarrollo (euros de investigación y desarrollo por euro de ingresos).

DisCDP $i t$, CDP para la información revelada en el cuestionario. Puntuación entre 0 y 100 puntos básicos.

A continuación se procede a analizar los resultados.

\section{Resultados}

En primer lugar, se calculan los estadísticos descriptivos para las variables de referencia para la medida del desempeño económico-financiero (ver Tabla 2). Como se puede observar, hay grandes diferencias en las variables $R O A$ y $R O E$, lo que se pone de manifiesto al constatar que la desviación típica es mayor que la media de la variable en ambos casos, no ocurriendo lo mismo en el caso de QT. Antes de realizar el análisis de regresión, obtenemos las correlaciones entre las variables de la muestra (ver Tabla 3). El objetivo que se persigue es analizar la existencia de relación, fortaleza y dirección de cada par de variables utilizadas en nuestro estudio. Como se esperaba, existe una correlación significativa entre las variables financieras debido a que en la composición de los indicadores que las constituyen intervienen las mismas variables. Así, por ejemplo, entre $R O A$ y $R O E$, el coeficiente de correlación de Pearson $(r=0,7876)$ señala que existe una relación fuerte y positiva puesto que las variaciones respecto a la media en $R O A$ se registran en el mismo sentido y con un elevado nivel de intensidad respecto a los producidos en el ROE. Del mismo modo, para las variables $R O A$ y $Q T$, el coeficiente $(r=0,1955)$ señala que existe una relación en la misma dirección entre ambos márgenes de resultados. Por su parte, existe también una correlación significativa, pero en sentido contrario entre $R O A$ y $T A(r=-0,1363)$; al igual que se observa entre $Q T$ y $T A(r=-0,4152)$. Con respecto a las variables de ecoeficiencia, se observa que hay evidencia de correlación significativa entre emisiones directas y variables económicas, como la que existe entre $E S$ y $R O A(\mathrm{r}=-0,1784)$. Del mismo modo, existe relación entre emisiones directas y variables financieras, como la correlación que se da entre $E S$ y $Q T(r=-0,2706)$. En ambos casos la dirección de la correlación es opuesta, lo cual indica que a menor valor en las emisiones, por euro facturado, mayores son los indicadores del DEc y la DFn.

Realizado el análisis de regresión (ver Tabla 4), los resultados confirman que la relación de la eco-eficiencia de los grupos empresariales analizados, en términos de emisiones, es estadísticamente significativa con el DEc y DFn, medidas por los márgenes $R O A$ y $R O E$, respectivamente, no sucediendo lo mismo con $Q T$. Los beneficios obtenidos en cuanto a la reducción de costes de producción, los incrementos de las ventas y reducción de los costes de financiación superan el coste de la amortización de las inversiones realizadas. También la mayor sensibilización respecto al impacto medioambiental puede estar influyendo en la sustitución de fuentes energéticas por otras que suponen menos emisiones. En este mismo sentido, la reorganización y mejora en los procesos de producción pueden estar contribuyendo a la reducción de las emisiones. 
Según lo anterior, podemos aceptar la hipótesis de que los grupos empresariales más proactivos con actuaciones medioambientales que conlleven mejores índices de ecoeficiencia en términos de emisiones directas, ES, sean las que mejor desempeño económico y financiero consigan, aunque, para el periodo y muestra analizados, no resulte ser premiada por los mercados de valores. Nuestro trabajo coincide con los resultados obtenidos previamente por Pogutz \& Russo (2009), Iwata \& Okada (2011), Busch \& Hoffman (2011) y Rokhmawati et al. (2015), y discrepa de otros como los de Konar \& Cohen (2001), Wagner et al. (2002), Sueyoshi \& Goto (2009) y Rassier \& Earnhart (2010).

Tabla 4

Resultados de los análisis de regresión

\begin{tabular}{lrrr}
\hline \multicolumn{1}{c}{$\begin{array}{c}\text { Variables } \\
\text { Dependientes }\end{array}$} & Modelo 1 ROA & Modelo 2 ROE & Modelo 3 QT \\
\hline ES $_{\text {it }}$ & $-2.3036^{* *}$ & $-7.0988^{* * *}$ & $5.3434^{* * *}$ \\
EILT $_{\text {it }}$ & -.0009 & -.0021 & .0003 \\
EIMT $_{\text {it }}$ & -.0175 & -.0693 & $.0154^{* *}$ \\
EIST $_{\text {it }}$ & $.3339^{* *}$ & .9742 & -.2101 \\
ROE $_{\text {it-1 }}$ & - & - & -.0366 \\
$\mathrm{TA}_{\text {it }}$ & -.6577 & -.0944 & -.6575 \\
$\mathrm{RD}_{\text {it }}$ & - & - & $2.7867^{* * *}$ \\
DisCDP $_{\text {it }}$ & - & - & $.0515^{* *}$ \\
\hline Obs. $_{\text {Breusch y Pagan Chi2 }}$ & $110.81^{* * *}$ & $68.46^{* * * *}$ & $50.91^{* * *}$ \\
Hausman Chi2 $^{*}$ & 2.41 & 1.28 & $28.69^{* * *}$ \\
$\mathrm{R}^{2}$ Wooldridge (AR1) F & $0.0185^{* * *}$ & $30.607^{* * *}$ & $6.994^{* * *}$ \\
\hline
\end{tabular}

Fuente: Elaboración Propia. Notas: ${ }^{*},{ }^{* *}$ y ${ }^{* *}$ nivel de significatividad del 10\%, 5\% y $1 \%$, respectivamente.

En cuanto a las variables de inversión ecoeficiente se observa el efecto positivo de la inversión a corto plazo (EIST) sobre los resultados económicos (ROA). Ya estudios como los de Nishitani et al. (2017) o Miroshnychenko et al. (2017) obtenían resultados similares aunque en ningún caso se distinguía el horizonte temporal al que se refería la inversión. Según los resultados obtenidos en este trabajo, los grupos empresariales pueden estar llevando a cabo gastos en actuaciones ecoeficientes a lo largo del ejercicio, hasta el volumen que sus resultados se lo permitan. La ausencia de relación positiva de las inversiones a medio y largo plazo, como se esperaba, queda argumentado porque esta inversión por parte del grupo sería de mayor riesgo financiero, ya que comprometen el resultado del ejercicio a futuro. Otra apreciación importante es que los grupos analizados divulgan información sobre su comportamiento ecoeficiente, sobre todo, referida a corto plazo, además de tratarse de la inversión más asequible y menos comprometedora para la estabilidad financiera del grupo.

Los mercados financieros (QT) recompensan a aquellos grupos empresariales con una inversión a medio plazo (EIMT) no siendo así para el corto ni el largo plazo (EIST, $E I L T)$. La nula relación entre la inversión a corto plazo y el valor del grupo empresarial puede atender a los distintos sectores que componen la muestra. Así, puede quedar explicado por la distinta penalización que realice el inversor en términos de riesgo para cada grupo o sector en particular durante ese ejercicio, independientemente de la variable rentabilidad económica o financiera. La inversión en actuaciones ecoeficientes a largo plazo puede que aún se perciba como un sobrecoste. Así, el uso de energía renovable o la adquisición de la tecnología necesaria para conseguir una mayor eficiencia en las emisiones aun no compensan en términos de coste-beneficio. Por lo anterior, el inversor no está dispuesto a apostar por ese grupo empresarial más allá de lo invertido a medio plazo.

Para el caso de las variables de control, los coeficientes de las variables que representan el tamaño empresarial (TA) no evidencian un impacto significativo sobre las variables que miden el desempeño económico o financiero, medido por el $R O A$ y $R O E$, respectivamente. Así pues, para la muestra y periodo seleccionado, los grupos empresariales de mayor tamaño no son las que menor rentabilidad y margen de beneficios obtienen. Para el caso del coeficiente de la variable de $R D$, se evidencia un impacto significativo y positivo sobre el $Q T$ poniendo de manifiesto que, en general, la inversión en I+D está siendo premiada por parte de los inversores coincidiendo con trabajos previos como los de Jaffe \& Palmer (1997) y Brunnermeier \& Cohen (2003). Del mismo modo, la variable DisCDP evidencia un impacto significativo y positivo sobre el $Q T$, puesto que la puntuación obtenida en la plataforma del cuestionario CDP puede repercutir positivamente sobre el beneficio esperado por los accionistas, en línea con estudios como los de Zamora-Ramírez, González-González \& Sabater-Marcos (2016). La rentabilidad obtenida por los grupos empresariales en el periodo anterior al de estudio no está influyendo en las expectativas de beneficio de los mercados de capitales $(Q T)$, no resultando significativa en el tercer modelo analizado.

\section{Conclusiones}

En la literatura previa se han encontrado muchos estudios que analizaron el efecto de los comportamientos ecoeficientes sobre el desempeño económico y financiero de las empresas, en particular de grandes grupos. Los resultados de estos trabajos han sido divergentes aunque, en los últimos años, son más los que demuestran una relación positiva respecto a los que no encuentran un efecto claro o negativo.

Este estudio aporta mayor evidencia en esta temática de la ecoeficiencia. Según los resultados obtenidos, una mejor ratio en ecoeficiencia, emisiones de $\mathrm{CO}_{2}$ respecto a euros facturados, se relaciona positivamente con el desempeño económico-financiero, medido mediante la rentabilidad sobre los activos y la rentabilidad sobre los fondos propios. También, sería recomendable llevar a cabo inversiones en actuaciones ecoeficientes a corto plazo ya que su efecto sobre la eficiencia en emisiones provocará la mejora de la rentabilidad económica.

En cuanto a los mercados financieros, los accionistas están recompensando las inversiones en ecoeficiencia a medio plazo, lo que podemos denominar como un compromiso semiestratégico de actuación medioambiental. Esta última conclusión estaría, en parte, en sintonía con lo que concluyen García-Sánchez et al. (2019) sobre la recompensa de los accionistas a las actitudes proactivas de las empresas por invertir en ecoinnovación y ecodiseño esperando un mayor retorno a largo plazo.

Aunque se demuestre una relación positiva entre un comportamiento ecoeficiente y el desempeño económicofinanciero, la realidad muestra que el ajuste necesario por parte de los grupos empresariales no es suficientemente oportuno. Para acelerar esa respuesta empresarial e incrementar las inversiones ecoeficientes los autores de este estudio opinan en la línea con las conclusiones de Porter \& Van der Linde (1995b). Así, una regulación medioambiental flexible, en cuanto a exigencias iniciales y con un plan de adaptación viable en plazos, conllevaría un aumento de iniciativas empresariales en cuanto a comportamientos más ecoeficientes. 
La propuesta anterior choca con la realidad debido a los pocos avances que se han producido en las últimas cumbres del clima. Los máximos responsables en el ámbito internacional no están dispuestos a incrementar las exigencias en sus respectivos países lo que retrasará la implantación de medidas que, a corto y medio plazo, reduzcan las emisiones de gases, a pesar de haber quedado demostrada su relación positiva con los resultados económicos-financieros de los grupos empresariales y el reconocimiento de los mercados a medio plazo.

En línea con lo anterior, también se necesita una respuesta más clara en cuanto a la recompensa por parte de los mercados de capitales. Así, los índices selectivos compuestos por los grupos empresariales más sensibilizados con su impacto sobre el cambio climático como es el DJSWI deberían añadir a sus exigentes criterios de inclusión, además de indicadores referidos a la cantidad y calidad de la información publicada, otros indicadores que muestren el retorno de ese comportamiento como, por ejemplo: ratios de ecoeficiencia, detalle de las inversiones realizadas con estimaciones de la reducción de emisiones o planes de viabilidad económica de la reducción de emisiones. También, asociado a la responsabilidad de los gestores, toda la información referida a los comportamientos ecoeficientes debería estar públicamente accesible y verificado, por el gran interés social que supone el cambio climático y el papel que tienen los grandes grupos cotizados líderes en sus respectivos sectores.

Otra contribución del estudio respecto a trabajos previos ha sido el empleo de una variable que cuantifica la inversión directa en ecoeficiencia, además de haber diferenciado el periodo de tiempo al que se refiere la inversión (corto, medio o largo plazo). En las investigaciones previas se han empleado los gastos de I+D como proxy a las inversiones llevadas a cabo en medidas de protección medioambiental. La mayor limitación de esta variable sería que, además de innovaciones cuyo objetivo es minorar el impacto sobre el medioambiente, puede estar recogiendo gastos asociados a otros objetivos muy distintos.

La principal limitación del presente estudio sería que analiza el comportamiento de grandes grupos empresariales cotizados. Lo anterior no permite generalizar los resultados para grupos no cotizados, pequeñas y medianas empresas que suponen otra parte importante de las emisiones de gases. Otra limitación es el número de grupos empresariales. Un mayor número de observaciones permitiría obtener unos resultados más robustos, segmentar la muestra por sectores según su nivel de impacto medioambiental, tamaños o por países.

\section{Financiación}

Esta investigación no recibió ninguna subvención específica de organismos/entidades de los sectores público, comercial o sin ánimo de lucro.

\section{Conflicto de intereses}

Los autores declaran no tener nignún conflicto de intereses.

\section{Referencias}

Adams, R., Jeanrenaud, S., Bessant, J., Denyer, D., \& Overy, P. (2016). Sustainability oriented innovation: a systematic review. International Journal of Management Reviews. 18 (2), 180-205. https://doi.org/10.1111/ijmr.12068

Al-Tuwaijri, S.A., Christensen, T.E., \& Hughes, K.E. (2004). The relations among environmental disclosure, environmental performance, and economic performance: A simultaneous equations approach. Accounting Organization Society, 29(5), 447-471. https://doi.org/10.1016/ s0361-3682(03)00032-1

Aragón-Correa, J.A., Hurtado-Torres, N., Sharma, S., \& García-Morales, V.J. (2008). Environmental strategy and performance in small firms: a resource-based perspective. Journal of Environmental Management, 86(1), 88-103. https://doi.org/10.1016/j.jenvman.2006.11.022

Arbelo, A., Pérez-Gómez, P., Rosa-González, F.M., \& Ramos, L. (2014). Eco-Efficiency: Environ 0 mental Performance Vs Economic Performance. Management Studies, 2(4), 239253.

Barbu, E. M., Dumontier, P., Feleag, N., \& Feleag, L. (2014), Mandatory environmental disclosures by companies complying with IASs/IFRSs: The cases of France, Germany, and the UK. The International Journal of Accounting, 49(2), 231-247. https://doi.org/10.1016/j.intacc. 2014.04.003

Bendixen, M., \& Abratt, R. (2007). Corporate identity, ethics and reputation in supplier-buyer relationships. Journal of Business Ethics, 76(1), 69-82. https://doi.org/10.1007/ s10551-006-9273-4

Blanco, C., Caro, F., \& Corbett, C. J. (2016). The state of supply chain carbon footprinting: analysis of CDP disclosures by US firms. Journal of Cleaner Production, 135, 1189-1197. https://doi.org/10.1016/j.jclepro. 2016.06.132.

Brunnermeier, S.B., \& Cohen, M.A. (2003). Determinants of environmental innovation in US manufacturing industries. Journal of Environmental Economics and Management, 45(2), 278-293. https://doi.org/10.1016/ s0095-0696(02)00058-X

Busch, T., \& Hoffmann, V.H. (2011). How Hot Is Your Bottom Line? Linking Carbon and Financial Performance. Business \& Society, 50(2), 233-265. https://doi.org/10.1177/ 0007650311398780

Butz, C., \& Plattner, A. (1999), Sustainable Share Investments: An Analysis of Returns Depending on Environmental and Social Criteria. Basel: Sarasin Sustainable Investment/Bank Sarasin.

Camisón-Zornoza, C., Lapiedra-Alcamí, R., SegarraCiprés, M., \& Boronat-Navarro, M. (2004). A meta-analysis of innovation and organizational size. Organization studies, 25(3), 331-361. https://doi.org/10.1177/0170840604040039

Delmas, M.A., \& Nairn-Birch, N.S. (2010). Is the Tail Wagging The Dog? An Empirical Analysis of Corporate Carbon Footprints and Financial Performance. California: UCLA.

European Union, EU (2009). Directive 2009/29/EC of the European parliament and of the council of 23 April 2009 amending directive 2003/87/EC so as to improve and extend the greenhouse gas emission allowance trading scheme of the community. Official Journal of the European Union. Available at https://eur-lex.europa.eu/legal-content/EN/TXT/PDF/ ?uri=CELEX:32009L0029\&from $=E N$

Feldman, S. J., Soyka, P.A., \& Ameer, P. (1997). Does improving a firm's environmental management system and environmental performance result in a higher stock price? The Journal of Investing, 6(4), 87-97. https://doi. 
org/10.3905/joi.1997.87

Fombrun, C., \& Shanley, M. (1990). What's in a name? Reputation building and corporate strategy. Academy of Management Journal, 33(2), 233-258. https://doi.org/10. $5465 / 256324$

Freeman, A. V., Myrick, A., \& Haveman, R. H. (1973). The Economics of Environmental Policy. New York: John Wiley.

Gallardo-Álvarez, D., \& Castilla-Polo, F. (2015). Modelo de gestión para la responsabilidad social en cooperativas. Revista Economía Industrial, (396), 139-149.

Gallego-Álvarez, I., Rodríguez-Domínguez, L., \& GarcíaSánchez, I. M. (2011). Study of some explanatory factors in the opportunities arising from climate change. Journal of Cleaner Production, 19(9-10), 912-926. https:// doi.org/10.1016/j.jclepro.2011.02.012

García-Sánchez, I.M., Gallego-Álvarez, I. and Zafra-López, J.L. (2019): Do the ecoinnovation and ecodesing strategies generate value added in munificent environments? Business Strategy and the Environment, https://doi.org/ 10.1002/bse.2414

González-Benito J., \& González-Benito, O. (2006). A review of determinant factors of environmental proactivity. Business Strategy and the Environment, 15(2): 87-102.

Hallegatte, S., Bangalore, M., Bonzanigo, L. F., M; Kane, T., Narloch, U., Rozenberg, J., Treguer, D., VogtSchilb, A. (2016). Shock Waves Managing the Impacts of Climate Change on Poverty. Available in https://openknowledge.worldbank.org/bitstream/ handle/10986/22787/9781464806735.pdf

Hart, S.L., \& Ahuja, G. (1996). Does it pay to be Green? An Empirical Examination of the Relationship between Emission reduction and Firm Performance. Business Strategy and the Environment, 5, 30-37. https://doi.org/10.1002/(sici)1099-0836(199603)5: 1\%3C30::aid-bse38\%3E3.3.co;2-h

Hatakeda, T., Kokubu, K., Kajiwara, T., \& Nishitani, K. (2012). Factors Influencing Corporate Environmental Protection Activities for Greenhouse Gas Emission Reductions: The Relationship between Environmental and Financial Performance. Environmental and Resource Economics, 53(4), 455-481. https://doi.org/10.1007/ s10640-012-9571-5

Horváthová, E. (2010). Does environmental performance affect financial performance? A meta-analysis. Ecological Economics, 70(1), 52-59. https://doi.org/10.1016/j. ecolecon.2010.04.004

Huppes, G., \& Ishikawa, M. (2005). Eco-efficiency and its terminology. Journal of Industrial Eco ology, 9(4), 43-46. https://doi.org/10.1162/108819805775247891

Intergovernmental Panel on Change Climate, IPCC (2018). Publications and data. Available in http://www.ipcc.ch/ publications_and_data/publications_and_data.shtml

International Civil Aviation Organization, IC̄CT. (2017). International Civil Aviation Organization Carbon Offset and Reduction Scheme for International Aviation (Corsia). Polici Update, pp. 1-7. Retrieved from http://www.theicct. org/sites/default/files/publications/ICAO MBM_PolicyUpdate 13022017_vF.pdf

Ittner, C. D., Larcker, D. F., \& Randall, T. (2003). Performance implications of strategic performance measurement in financial services firms. Accounting, Organizations and Society, 28(7-8), 715-741. https://doi.org/10.1016/ S0361-3682(03)00033-3

Iwata, H., \& Okada, K. (2011). How Does Environmental Performance Affect Financial Performance? Eviden- ce from Japanese Manufacturing Firms. Ecological Economics, 70(9), 1691-1700. https://doi.org/10.1016/j. ecolecon.2011.05.010

Jaffe, A.B., \& Palmer, K. (1997). Environmental regulation and innovation: A panel data study. Review of Economics and Statistics, 79(4), 610-619. https://doi.org/10.1016/ j.ecolecon.2011.05.010

Kemp, R. (1997). Environmental Policy and Technical Change, Edward Elgar Publishing, number 1187, April.

King, A., \& Lenox, M. (2001). Does it Really Pay to be Green? Accounting for Strategy Selection in the relationship between environmental and Financial Performance. Journal of Industrial Ecology, 4, 105-116. https://doi.org/10. 1162/108819801753358526

King, A., \& Lenox, M.J. (2002). Exploring the locus of profitable pollution reduction. Management Science, 48(2), 289-299. https://doi.org/10.1287/mnsc.48.2.289.258

Kneller, R., \& Manderson, E., (2012). Environmental regulations and innovation activity in UK manufacturing industries. Resource. Energy Economy, 34 (2), 211-235. https://doi.org/10.1016/j.reseneeco.2011.12.001

Knox, S., \& Maklan, S. (2004). Corporate social responsibility: Moving beyond investment towards measuring outcomes. European Management Journal, 22(5), 508-516. https://doi.org/10.1016/j.emj.2004.09.009

Konar, S., \& Cohen, M.A. (2001). Does the market value environmental performance? The Review of Economics and Statistics, 83(2), 281-289. https://doi.org/10.1162/ 00346530151143815

La Rosa, F., Liberatore, G., Mazzi, F., \& Terzani, S. (2018). The impact of corporate social performance on the cost of debt y access to debt financing for listed European nonfinancial firms. European Management Journal, 36(4), 519-529. https://doi.org/10.1016/j.emj.2017.09.007

Lankoski, L. (2006). Differential economic impacts of corporate responsibility issues. Business \& Society, 48(2), 206224. https://doi.org/10.1177/0007650307306635

Lothe, S., Myrtveit, I., \& Trapani, T. (1999). Compensation systems for improving environmental performance. Business Strategy and the Environment, 8(6), 313321. https://doi.org/10.1002/(sici)1099-0836(199911/ 12) 8:6\%3C313::aid-bse219\%3E3.3.co;2-3

Majumdar, S.K., \& Marcus, A.A. (2001). Rules versus discretion: the productivity consequences of flexible regulation. Academy of Management Journal, 44(1), 170-179. https://doi.org/10.5465/3069344

Miroshnychenko, I., Barontini, R., Testa, F. (2017). Green practices and financial performance: A global outlook. Journal of Cleaner Production, 147, 340-351. https://doi.org/10.1016/j.jclepro.2017.01.058

Misani, N., \& Pogutz, S. (2015). Unraveling the effects of environmental outcomes and processes on financial performance: A non-linear approach. Ecological economics, 109, 150-160. https://doi.org/10.1016/j.ecolecon. 2014.11.010

Molina-Azorín, J.F., Claver-Cortés, E., López-Gamero, M.D., \& Tarí, J.J. (2009). Green management and financial performance: A literature review. Management Decision, 47(7), 1080-1100. https://doi.org/10.1108/ 00251740910978313

Müller, K., \& Sturm, A. (2001). Standartized Eco-efficiency Indicators. Basel, Switzerland: Ellip oson AG.

Nakao, Y., Amano, A., Matsumura, K., Genba, K., \& Nakano, M. (2007). Relationship between environmental performance and financial performance: an empirical analysis of Japanese corporations. Business Strategy and the Envi- 
ronment, 16(2), 106-118. https://doi.org/10.1002/bse. 476

Nishitani, K., Jannah, N., Kaneko, H. (2017). Does corporate environmental performance enhance financial performance? An empirical study of Indonesian firms. Environmental Development, 23, 10-21. https://doi.org/10. 1016/j.envdev.2017.06.003

Nishitani, K., Kaneko, S., Fujii, H., \& Komatsu, S., (2011). Effects of the reduction of pollution emissions on the economic performance of firms: an empirical analysis focusing on demand and productivity. Journal of Cleaner Production, 19(17-18), 1956-1964. https://doi.org/10.1016/j. jclepro.2011.06.021

Pérez-Calderón, E., Milanés-Montero, P., \& Ortega-Rossell, F. J. (2012). Environmental performance and firm value: Evidence from Dow Jones Sustainability Index Europe. International Journal of Environmental Research, 6(4), 1007-1014. https://ijer.ut.ac.ir/article 571.html

Pogutz, S., \& Russo, A. (2009). Eco-efficiency vs Ecoeffectiveness. Exploring the link between GHG emissions and firm performance. Academy of Management Proceedings, 1, 1-6. https://doi.org/10.2139/ssrn.1467790

Pomering, A., \& Johnson, L.W. (2009). Constructing a corporate social responsibility reputation using corporate image advertising. Australasian Marketing Journal, 17 (2), 106-114. https://doi.org/10.1016/j.ausmj.2009.05.006

Porter, M. E. (1991). America's green strategy. Scientific American, 264(4), 168-179.

Porter, M. E., \& Van der Linde, C. (1995a). Green and competitive: Ending the stalemate. Harvard Business Review, 73(5), 120-134.

Porter, M. E., \& Van der Linde, C. (1995b). Toward a new conception of the environment competitiveness relationship. Journal of economic perspectives, 9 (4), 97-118. https: //doi.org/10.1257/jep.9.4.97

Prado-Lorenzo, J.M., Rodriguez-Dominguez, L., GallegoÁlvarez, I., \& García-Sánchez, I.M. (2009). Factors influencing the disclosure of greenhouse gas emissions in companies world-wide. Management Decision, 47 (7), 1133-57. https://doi.org/10.1108/ 00251740910978340

Przychodzen, J., \& Przychodzen, W. (2015). Relationships between eco-innovation and financial performanceevidence from publicly traded companies in Poland and Hungary. Journal of Cleaner Production, 90, 253-263. https://doi.org/10.1016/j.jclepro.2014.11.034

Rahmand, N. R. A., Rasid, S. Z. A., \& Basiruddin, R. (2014). Exploring the Relationship between Carbon Performance, Carbon Reporting and Firm Performance: A Conceptual Paper. Procedia-Social and Behavioural Sciences, 164(31), 118-125. https://doi.org/10.1016/j.sbspro. 2014.11.059

Rassier, D.G., \& Earnhart, D. (2010). Does the Porter hypothesis explain expected future financial performance? The effect of clean water regulation on chemical manufacturing firms. Environmental and Resource Economics, 45(3), 353-377. https://doi.org/10.1007/s10640-009-9318-0

Rokhmawati, A., Sathye, M., \& Sathye, S. (2015). The Effect of GHG Emission, Environmental Performance, and Social Performance on Financial Performance of Listed Manufacturing Firms in Indonesia. Procedia-Social and Behavioral Sciences, 211, 461-470. https://doi.org/10.1016/ j.sbspro.2015.11.061

Rubashkina, Y., Galeotti, M., \& Verdolini, E., (2015). Environmental regulation andcompetitiveness: empirical evidence on the porter hypothesis from European manufactu- ring sectors. Energy Policy, 83, 288-300. https://doi.org/ $10.2139 /$ ssrn. 2463538

Russo, M.V., \& Fouts, P.A. (1997). A Resource Based Perspective on Corporate Environmental Performance and Profitability. Academy of Management Journal, 40(3), 534-559. https://doi.org/10.5465/257052

Schmidheiny, S. (1992). Chan oging Course: A Global Business Perspective on Development and the Environment. Cabridge, Mass: MIT Press.

Sprinkle, G.B., \& Maines, L.A. (2010). The benefits and costs of corporate social responsibility. Business Horizons, 53 (5), 445-453. https://doi.org/10.1016/j.bushor.2010.05. 006

Stubbs, W., \& Cocklin, C. (2008). Conceptualizing a "Sustainability business model. Organization \& environment. 21 (2), 103-127. https://doi.org/10.1177/ 1086026608318042

Sueyoshi, T., \& Goto M. (2009). Can environmental investment and expenditure enhance financial performance of US electric utility firms under the clean air act amendment of 1990? Energy Policy, 37(11), 4819-4826. https: //doi.org/10.1016/j.enpol.2009.06.038

Teece, D.J., (2010). Business models, business strategy and innovation. Long Range Planning, 43(2-3), 172-194. https://doi.org/10.1016/j.1rp.2009.07.003

Thomas, A. (2001). Corporate Environmental Policy and Abnormal Stock Price Returns: An Empirical Investigation. Business strategy and the Environment, 10, 125-134. https://doi.org/10.1002/bse.281

United Nations Framework Convention on Climate Change, UNFCCC (2016). Paris Agreement, https://unfccc.int/files/meetings/paris/_nov/_2015/ application/pdf/paris/_agreement/_english/_.pdf.

Verfaillie, H., \& Bidwell, R. (2000). Measuring eco-efficiency: A guide to reporting company performance. London, UK: World Business Council of Sustainable Development.

Wagner, M. (2005). How to reconcile environmental and economic performance to improve corporate sustainability: corporate environmental strategies in the European paper industry. Journal of Environmental Management, 76, 105118. https://doi.org/10.1016/j.jenvman.2004.11.021

Wagner, M. (2015). The link of environmental and economic performance: Drivers and limitations of sustainability integration. Journal of Business Research, 68(6), 13061317. https://doi.org/10.1016/j.jbusres.2014.11.051

Wagner, M., Van Phu, N., Azomahou, T., \& Wehrmeyer, W. (2002). The relationship between the environmental and economic performance of firms: an empirical analysis of the European paper industry. Corporate SocialResponsibility and Environmental Management, 9(3), 133-146. https://doi.org/10.1002/csr.22WBCSD, World Business Council for Sustainable Development, (1997). Environmental Perfor ${ }^{\circ}$ mance and Shareholders Value. WBCSD: Conches-Geneva.

WBCSD, World Business Council for Sustainable Development, (2000). Eco-Efficiency: Creating more value with less impact. WBCSD: Conches-Geneva.

Weinhofer, G., \& Hoffmann, V., (2010). Mitigating climate change e how do corporate strategies differ? Business Strategy and the Environment, 19, 77-89. https://doi. org/10.1002/bse.618

Yang, L., \& Zhang, X. (2018). Assessing regional ecoefficiency from the perspective of resource, environmental and economic performance in China: a bootstrapping approach in global data envelopment analysis. Journal 
of Cleaner Production, 173, 100-111. https://doi.org/10. 1002/bse.618

Zamora-Ramírez, C., González-González, J.M., \& SabaterMarcos, A. (2016). Carbon reporting: Analysis of the Spanish market response. Revista Española de Financiación y Contabilidad, 45(2), 231-265. https://doi.org/10.1080/ 02102412.2016 .1162972

Ziegler, A., \& Schröder, M. (2010). What determines the inclusion in a sustainability stock index?. A panel data analysis for european firms. Ecological Economics, 69(4), 848-856. https://doi.org/10.1016/j.ecolecon.2009.10.009 